\title{
Neuroradiologic Diagnosis of Minor Leak prior to Major SAH: Diagnosis by T1-FLAIR Mismatch
}

\author{
S. Oda, M. Shimoda, A. Hirayama, M. Imai, F. Komatsu, H. Shigematsu, J. Nishiyama, and M. Matsumae
}

\begin{abstract}
BACKGROUND AND PURPOSE: In major SAH, the only method to diagnose a preceding minor leak is to ascertain the presence of a warning headache by interview; however, poor clinical condition and recall bias can cause inaccuracy. We devised a neuroradiologic method to diagnose previous minor leak in patients with SAH and attempted to determine whether warning (sentinel) headaches were associated with minor leaks before major SAH.
\end{abstract}

MATERIALS AND METHODS: We retrospectively evaluated 127 patients who were admitted with SAH within 48 hours of ictus. Previous minor leak before major SAH was defined as TIWI-detected clearly bright hyperintense subarachnoid blood accompanied by SAH blood on FLAIR images that was distributed over a larger area than bright hyperintense subarachnoid blood on TIWI (TI-FLAIR mismatch).

RESULTS: The incidence of warning headache before SAH was $11.0 \%$ (14 of 127 patients, determined by interview). The incidence of TT-FLAIR mismatch (neuroradiologic diagnosis of minor leak before major SAH) was 33.9\% (43 of 127 patients). Of the 14 patients with warning headache, 13 had a minor leak diagnosed by T1-FLAIR mismatch at the time of admission. Variables identified by multivariate analysis as significantly associated with minor leak diagnosed by T1-FLAIR mismatch included 80 years of age or older, rebleeding after admission, intracerebral hemorrhage on $\mathrm{CT}$, and mRS scores of 3-6.

CONCLUSIONS: We conclude that warning headaches diagnosed by interview are not a product of recall bias but are the result of actual leaks from aneurysms.

ABBREVIATIONS: BHSB = bright hyperintense subarachnoid blood; $I \mathrm{CH}=$ intracerebral hemorrhage

A severe, sudden headache, known as a warning or sentinel headache, sometimes presents during the days or weeks before aneurysmal SAH. ${ }^{1,2}$ These warning headaches have been interpreted as reflecting a minor or warning leak that arises from the first small bleed from an aneurysm before a major SAH. ${ }^{3,4}$ However, in patients who have had major SAH, the presence of a warning headache can only be determined by interview and relies on recall, and there are no established neuroradiologic diagnostic procedures to prove the existence of a previous minor leak. Accurate diagnosis of previous warning headaches by interview is difficult because of the inability to obtain complete information from patients in poor clinical

Received December 11, 2014; accepted after revision February 9, 2015.

From the Department of Neurosurgery (S.O., M.S., A.H., M.I., F.K., H.S., J.N.), Tokai University Hachioji Hospital, Tokyo, Japan; and Department of Neurosurgery (M.M.), Tokai University School of Medicine, Kanagawa, Japan.

Please address correspondence to Masami Shimoda, MD, Department of Neurosurgery, Tokai University Hachioji Hospital, 1838 Ishikawa-machi, Hachioji, Tokyo

192-0032, Japan; e-mail: mashimoda-nsu@umin.ac.jp

http://dx.doi.org/10.3174/ajnr.A4325 conditions. One report states that warning headaches are innocuous and unrelated to SAH because an alternative explanation of warning headache is recall bias. ${ }^{5}$

In the subacute phase of SAH (>3-4 days after onset), T1WI depicts subarachnoid blood as high-signal-intensity areas. FLAIR images depict subarachnoid blood as high-signal-intensity areas from the acute-to-subacute phase of SAH. In patients with SAH with T1-detected clearly bright hyperintense subarachnoid blood (BHSB), we proposed a "T1-FLAIR mismatch" to define patients who had experienced a prior minor leak: BHSB on T1WI obtained at admission was considered subacute and FLAIR hyperintense blood that was more extensive than this was considered acute (with the T1 clearly BHSB component defined as the subacute minor leak). Using this diagnostic procedure, we investigated the association between warning headaches and what was considered a minor leak on MR imaging (ie, the first SAH, due to a ruptured aneurysm that occurred before major SAH). Furthermore, we studied the clinical features of patients with SAH with a neuroradiologically diagnosed minor leak before major SAH. 


\section{MATERIALS AND METHODS}

\section{Patient Population}

Subjects were selected from the 210 patients in the nontraumatic aneurysmal acute-phase SAH data base at our hospital between September 2002 and May 2014. Of these patients, 12 receiving conservative therapy instead of surgery because of the absence of brain stem reflexes and 12 with SAH due to ruptured dissecting aneurysms were excluded from this study. The remaining 186 patients underwent acute-stage aneurysmal surgery by craniotomy or an interventional procedure within 72 hours of SAH onset. From these 186 patients, we identified 127 who were admitted within 48 hours of SAH onset and underwent adequate MR imaging, including DWI, FLAIR imaging, T1WI, and CT, on admission. These 127 patients with SAH had stable vital signs reflecting adequate respiration and circulation and underwent serial MR imaging and CT after admission. All MR imaging studies were preceded by the procurement of informed consent by the patient and/or their relative. Initial MR imaging on admission was performed before conventional angiographic, surgical, or endovascular procedures in all cases.

Clinical and imaging records were evaluated retrospectively. Study approval was obtained from the Institutional Review Board for Clinical Research at our university.

\section{Imaging Protocol}

For patients with aneurysmal SAH, we routinely performed serial MR imaging on admission, within 2-7 days after surgery, and before patients were discharged. Serial MR imaging was performed for the evaluation of primary brain damage, surgical complications, and ischemic lesions due to vasospasm. At all time points, serial MR imaging included axial conventional T1WI, FLAIR, DWI, and MRA and was performed by using a $1.5 \mathrm{~T}$ superconducting magnet (Signa Excite or HDx; GE Healthcare, Milwaukee, Wisconsin) with a quadrature head coil. Pulse sequences were as follows: FLAIR (TR/TE, 8000/120 ms; TI, $2000 \mathrm{~ms}$; section thickness/section gap, 7.0/1.0 mm; FOV, $24 \times 24 \mathrm{~cm}$; NEX, 1; matrix, $256 \times 224)$, T1WI (TR/TE, 2000/24 ms; TI, $750 \mathrm{~ms}$; section thickness/section gap, 7.0/1.0 mm; FOV, $24 \times$ $24 \mathrm{~cm}$; NEX, 2; matrix, $256 \times 192$ ), and 3D time-of-flight MRA (TR/TE, 27/6.8 ms; flip angle, $16^{\circ}$; bandwidth, $14.7 \mathrm{~Hz}$; FOV, $18 \times 18 \mathrm{~cm}$; slab thickness, $70 \mathrm{~mm}$; section thickness, $1.2 \mathrm{~mm}$; matrix $256 \times 192$; NEX, 1). MR imaging was completed within 13-15 minutes. Contrast material such as gadolinium was not used in this study. In the evaluation of FLAIR images, we carefully distinguished the disease or pathologic condition accompanied by hyperintensity in the subarachnoid space on FLAIR images from artifacts such as CSF pulsation or metallic artifacts, infectious meningitis, and leptomeningeal metastasis or anastomosis. For the subjects in this study, we did not perform highly concentrated oxygen inhalation before or at the time of the MR imaging.

Plain CT scans were obtained in all patients in whom SAH was diagnosed by MR imaging on admission. The CT section thickness was $4 \mathrm{~mm}$. 3D CTA was performed in all patients to identify ruptured aneurysms and estimate major-vessel early vasospasm.

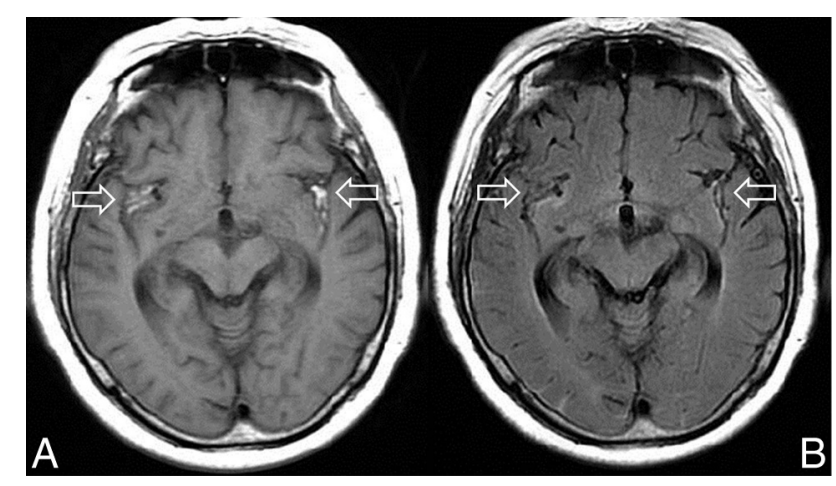

FIG 1. A 77-year-old woman with a ruptured right middle cerebral artery aneurysm who was admitted 5 days after onset. TIWI on admission shows BHSB in the bilateral Sylvian fissure ( $A$, arrows) that matches the blood distribution on the FLAIR image ( $B$, arrows). These findings are typical of subacute SAH with T1-FLAIR-matched SAH that was not associated with rebleeding. Reprinted with permission from Shimoda M. Neuroimaging for headache. Journal of Clinical and Experimental Medicine (IGAKU NO AYUMI) 2012;243:1086-94; Ishiyaku Publishers, Inc.

\section{Definition of Variables}

The clinical grade of each patient was determined on admission according to the World Federation of Neurological Surgeons grading system. ${ }^{6}$ The severity of SAH was classified by using CT findings according to the Fisher scale. ${ }^{7}$ Warning headache was defined as a sudden and unusually severe headache of at least 1 hour followed by a symptom-free interval or marked improvement of symptoms before admission due to major SAH. The presence of a warning headache was diagnosed by a thorough interview of patients and their relatives or accompanying persons.

Among patients with T1WI-detected clearly BHSB on admission MR imaging, if the distribution of SAH on T1 (T1 BHSB component) almost entirely matched the distribution of SAH on FLAIR (FLAIR hyperintense component), patients were diagnosed with subacute SAH not associated with rebleeding and were thus excluded from the study (Fig 1). In the remaining patients with T1WI-detected BHSB, if the subarachnoid blood on FLAIR images was distributed over a larger area than the BHSB on T1WI (T1-FLAIR mismatch), this finding was taken as an indication of new, acute SAH. T1WI-detected BHSB in these patients represents subacute SAH due to a previous minor leak (Fig 2). If FLAIR-detected SAH had no T1-detected BHSB on admission MR imaging, SAH was considered acute.

Diagnosis of intracerebral hemorrhage ( $\mathrm{ICH}$ ) was defined as a blood collection with a diameter of $>1 \mathrm{~cm}$ detected by CT. Large intra-Sylvian hematoma was based on the presence of clots of $>5$ $\mathrm{mL}$. Rebleeding after admission was defined as a definite increase in the volume of blood visible on CT accompanied by sudden exacerbation of consciousness compared with baseline MR imaging or CT on admission. Delayed ischemic neurologic deficits were defined as the appearance of a focal neurologic abnormality following recovery from the immediate postoperative state. CT and MR imaging findings were interpreted by at least 2 senior stroke neurosurgeons (M.S. and S.O.), with 32 and 27 years of experience, respectively. In the case of disagreement between raters, diagnosis was obtained by consensus.

Outcome was assessed at 3 months by using the mRS. ${ }^{8} \mathrm{~Pa}-$ AJNR Am J Neuroradiol 36:1616-22 Sep 2015 www.ajnr.org 1617 


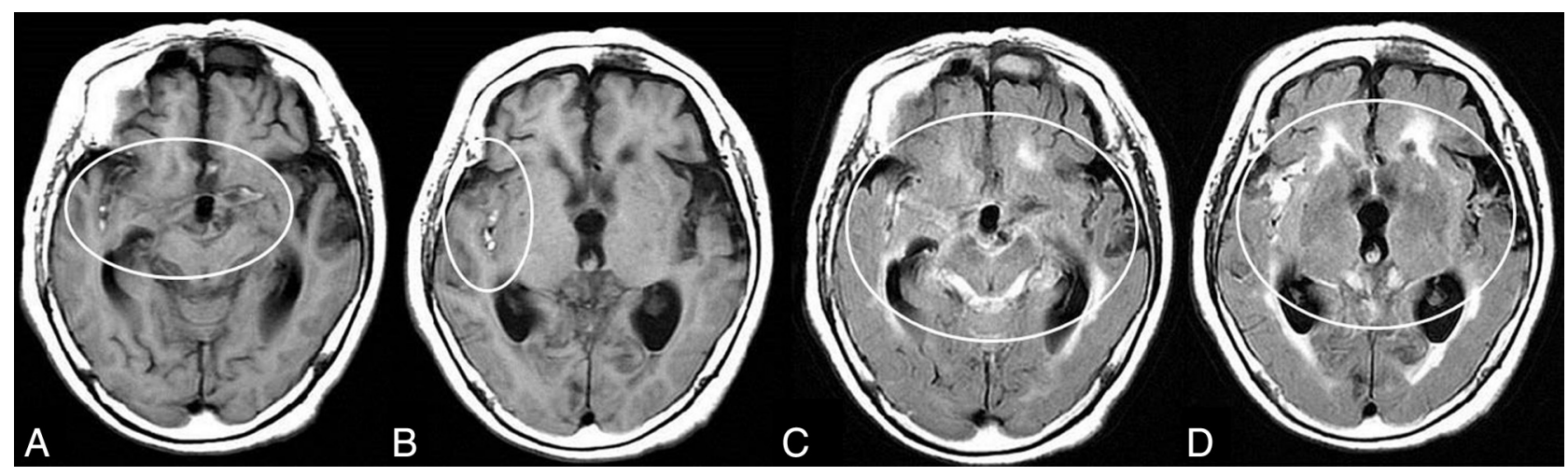

FIG 2. Typical neuroradiologic findings in a patient with minor leak before major SAH attack. Images are from an 80-year-old woman with a ruptured right middle cerebral artery aneurysm. TIWI on admission shows clearly iso- to mildly hyperintense blood obscuring the right Sylvian fissure, in addition to the more conspicuous bright $T\rceil$ foci $(A$ and $B$, circles). These findings indicate subacute subarachnoid blood due to a minor leak that occurred before the major attack. FLAIR images on admission show the SAH in the acute phase in the quadrigeminal cistern and left Sylvian fissure ( $C$ and $D$, circles), in addition to the BHSB on TIWI. We defined this as TI-FLAIR mismatch and used it as a neuroradiologic diagnosis of minor leak that occurred before major SAH.

Table 1: Clinical features, neurologic status, and CT features on admission ${ }^{\mathrm{a}}$

\begin{tabular}{|c|c|c|c|c|}
\hline & \multirow[b]{2}{*}{ Total } & \multicolumn{2}{|c|}{ T1-FLAIR Mismatch } & \multirow[b]{2}{*}{$P$ Value } \\
\hline & & Positive & Negative & \\
\hline No. of patients & 127 & $43(33.9)$ & $84(66.1)$ & \\
\hline \multicolumn{5}{|l|}{ Warning sign } \\
\hline Positive & 14 & $13(30.2)$ & $1(1.2)$ & $<.001$ \\
\hline Negative & 54 & $2(4.7)$ & $52(61.9)$ & \\
\hline Unknown & 59 & $28(65.1)$ & $31(36.9)$ & \\
\hline Mean age (yr) & $61.5 \pm 14.2$ & $66.5 \pm 13.2$ & $58.9 \pm 14.0$ & .004 \\
\hline Age range (yr) & 21-89 & $40-85$ & 21-89 & \\
\hline \multicolumn{5}{|l|}{ Elderly patients } \\
\hline Older than 80 years & 12 & $10(23.3)$ & $2(2.4)$ & $<.001$ \\
\hline Female sex & 89 & $32(74.4)$ & $57(67.9)$ & .541 \\
\hline Rebleeding after admission & 28 & $19(44.2)$ & $9(1.7)$ & $<.001$ \\
\hline \multicolumn{5}{|l|}{ WFNS grade on admission } \\
\hline Grades I-II & 97 & $28(65.1)$ & $69(82.1)$ & .028 \\
\hline Grades IV-V & 28 & $13(30.2)$ & 15 (17.9) & .120 \\
\hline \multicolumn{5}{|l|}{ Fisher group } \\
\hline Group 3 & 85 & $30(69.8)$ & $55(65.5)$ & .550 \\
\hline Intracerebral hemorrhage & 25 & $19(44.2)$ & $6(7.1)$ & $<.001$ \\
\hline Acute hydrocephalus & 61 & $26(60.5)$ & $35(41.7)$ & .060 \\
\hline \multicolumn{5}{|l|}{ Aneurysm site } \\
\hline Anterior communicating artery & 41 & $9(20.9)$ & $32(38.1)$ & \\
\hline Anterior cerebral artery & 8 & $4(9.3)$ & $4(4.8)$ & \\
\hline Internal carotid artery & 38 & $12(27.9)$ & $26(31.0)$ & \\
\hline Middle cerebral artery & 31 & $17(39.5)$ & $14(16.7)$ & \\
\hline Posterior circulation & 8 & 0 & $8(9.5)$ & .051 \\
\hline \multicolumn{5}{|l|}{ Aneurysm size } \\
\hline$>5 \mathrm{~mm}$ & 75 & $31(72.1)$ & $44(52.4)$ & .037 \\
\hline$>10 \mathrm{~mm}$ & 8 & $3(7.0)$ & $5(6.0)$ & 1.000 \\
\hline
\end{tabular}

Note:-WFNS indicates World Federation of Neurological Surgeons.

a Values are No. (\%) unless otherwise stated. The "No. of patients" row shows the percentage of the total number of patients, whereas all other percentages in the "Positive" and "Negative" columns are the percentages of patients with positive and negative findings, respectively.

tients were stratified into favorable outcome (mRS score of $0-2$ ) and unfavorable outcome (mRS score of 3-6). Postoperative management was administered according to previously reported protocols. ${ }^{9}$

\section{Statistical Analysis}

The significance of clinical factors potentially associated with minor leak before major attack diagnosed by T1-FLAIR mismatch on admission was determined by the Fisher exact test for categoric variables and an independent sample 2-tailed Student $t$ test for continuous variables. Clinical factors with a significance level of $P<.05$ were subjected to multivariate logistic regression analysis with the occurrence of minor leak diagnosed by T1-FLAIR mismatch on admission as the dependent variable. Statistical analyses were performed by using commercially available software (SPSS, Version 22.0 for Windows; IBM, Armonk, New York).

\section{RESULTS \\ Warning Headache and TI-FLAIR Mismatch}

The incidence of warning headache determined by interview was $11.0 \%$ (14/ 127 patients). Fifty-four patients $(42.5 \%)$ reported no history of warning headache before admission, and the presence or absence of warning headache was unknown in the remaining 59 patients (Table 1). Among the 14 patients who had a warning headache before the major attack, 12 were younger than 80 years of age.

Adverse events, such as neurologic deterioration or rebleeding from the aneurysm during or immediately after MR imaging did not occur in any patient. According to neuroradiologic diagnosis by using T1-FLAIR mismatch, the overall incidence of minor leak before major SAH was $33.9 \%$ (43/127 patients) (Table 1). Of the 14 patients with a history of warning headache before admission, 13 had previous minor leak diagnosed by T1-FLAIR mismatch on admission. In the remaining patient, the diagnosis of minor leak by T1-FLAIR mismatch was not possible because major SAH developed within 48 hours of the warning headache (minor leak) onset. In the 113 patients without a history of warning headache before admission, 
$30(23.6 \%)$ had T1-FLAIR mismatch on admission and therefore had a potential minor leak before admission diagnosed by T1FLAIR mismatch.

\section{Pre- and Postoperative Clinical Factors and TI-FLAIR Mismatch}

Tables 1 and 2 summarize the pre- and postoperative clinical factors that were significantly different between patients with and without T1-FLAIR mismatch on admission. Two patients had a major vessel vasospasm detected by 3D CTA or conventional digital subtraction angiography on admission. Both patients had a minor leak diagnosed by T1-FLAIR mismatch. In 35 of the 43 patients with T1-FLAIR mismatch on admission (81.4\%), MRA findings were indistinct due to subacute subarachnoid blood caused by the minor leak, particularly in the vessel around the ruptured aneurysm. In the 8 patients with minor leak before admission and without obfuscation of the visibility on MRA, subarachnoid clots on T1WI were small.

In all patients with T1-FLAIR mismatch and ICH, the highintensity signal on T1WI was present in the cistern around the ruptured aneurysm, and ICH was depicted as a high-intensity signal on FLAIR images (Fig 3).

Ten of the 12 elderly patients with SAH ( 80 years of age or older) had a minor leak diagnosed by T1-FLAIR mismatch. In 9 of these 10 elderly patients with T1-FLAIR mismatch, the presence or absence of a warning headache could not be determined by interview due to poor clinical condition.

Multivariate stepwise logistic regression analysis revealed that 80 years of age or older, the presence of rebleeding after admission, ICH on CT, and mRS scores of 3-6 were significantly associated with the diagnosis of minor leak by T1-FLAIR mismatch on admission (Table 3).

\section{DISCUSSION}

\section{Incidence of Minor Leak in Previous Reports}

According to Jakobsson et al, ${ }^{1}$ the reported incidence of warning signs in patients with SAH ranges from $13.5 \%$ to $60 \%$. They emphasized that the true incidence of warning signs is difficult to establish because it is difficult or impossible to obtain complete information from patients in poor clinical condition or who die before reaching a hospital. ${ }^{1}$ In addition to headaches, a previous study noted several nonspecific symptoms as warning signs, ${ }^{10}$ the incidences of which greatly differed. ${ }^{1}$ Thus, the diagnosis of a minor leak by interview has poor accuracy, and it is difficult to grasp the clinical significance of an interviewdiagnosed minor leak.

Our results revealed that 13 of 14 patients with a history of warning headache before admission had a minor leak before the major attack, as indicated by neuroradiologic images on admission. Therefore, we propose that warning headaches diagnosed by interview are not a product of recall bias but are the result of actual leaks from aneurysms (ie, a first SAH). In the future, neuroradiologic diagnosis could be used to investigate the clinical significance of minor leaks that cannot be diagnosed by interview.

\section{Neuroradiologic Diagnosis of Minor Leak before Major Attack}

The $\mathrm{T} 1$ relaxation time of acute subarachnoid blood is relatively shorter
"The "No. of patients" row shows the percentage of the total number of patients, whereas all other percentages in the "Positive" and "Negative" columns are the percentages of patients with positive and negative findings, respectively. ${ }^{\mathrm{b}}$ The incidence of chronic hydrocephalus was calculated for the surviving patients.

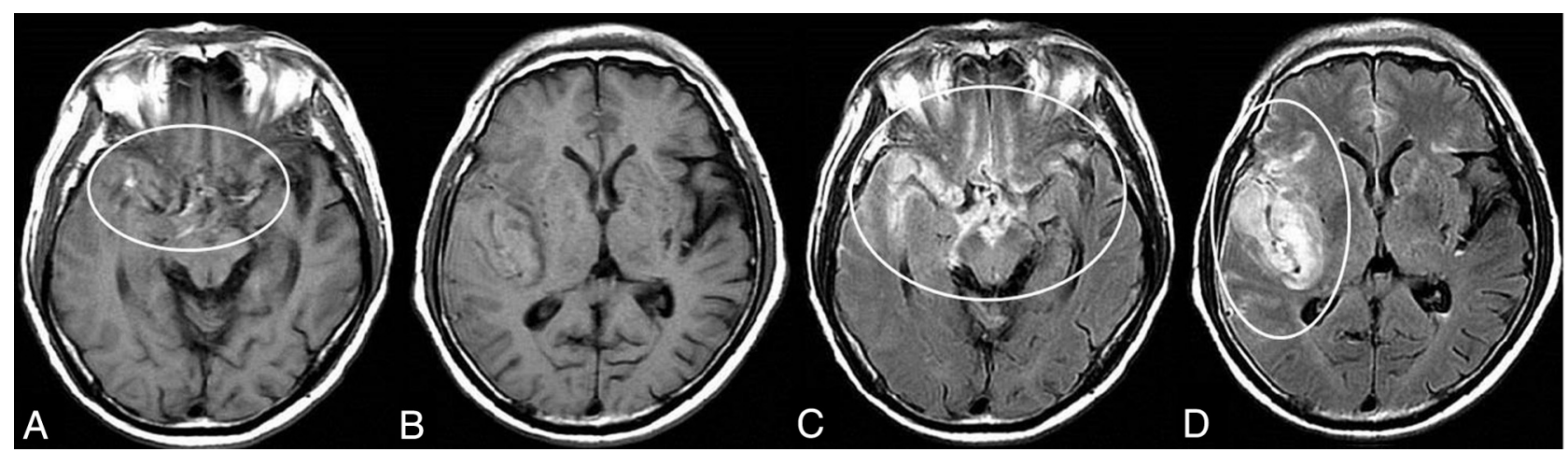

FIG 3. Typical neuroradiologic findings in a case with minor leak before a major SAH attack with intra-Sylvian hematoma. Images are from a 66-year-old woman with a ruptured right middle cerebral artery aneurysm. TTWI shows iso- to mildly hyperintense blood obscuring the right Sylvian fissure and suprasellar cistern, in addition to the more conspicuous bright $\mathrm{Tl}$ foci including the neighboring area of the ruptured aneurysm. An intra-Sylvian hematoma is depicted as an isointense signal (A circle, B). FLAIR images show the intra-Sylvian hematoma and SAH (right Sylvian fissure, suprasellar and right ambient cistern) as a high-intensity signal, which indicates acute blood ( $C$ and $D$, circles). 


\begin{tabular}{|c|c|c|c|}
\hline & Odds Ratio & $95 \% \mathrm{Cl}$ & $P$ Value \\
\hline \multicolumn{4}{|l|}{ Preoperative clinical factors } \\
\hline Age older than 80 years & 8.475 & $1.639-43.478$ & .011 \\
\hline Rebleeding & 5.291 & $2.028-13.889$ & .001 \\
\hline \multicolumn{4}{|l|}{$\begin{array}{l}\text { Associated neuroradiologic } \\
\text { findings on admission }\end{array}$} \\
\hline $\begin{array}{l}\text { Intracerebral hemorrhage } \\
\text { on CT }\end{array}$ & 7.197 & $2.457-20.833$ & $<.001$ \\
\hline Postoperative factors & & & \\
\hline mRS score 3-6 & 6.690 & $2.548-17.564$ & $<.001$ \\
\hline
\end{tabular}

than that of normal CSF. ${ }^{11,12}$ However, T1WI is not useful for the diagnosis of SAH in the acute phase because the degree of T1 shortening replaces normally black CSF space with isointensity. Beyond 4 days after SAH onset, conversion of oxyhemoglobin to methemoglobin increases gradually. ${ }^{13,14}$ Methemoglobin is a paramagnetic substance that causes substantial T1 shortening. Therefore, methemoglobin accumulation causes $\mathrm{T} 1$ shortening in the subacute and chronic phases, ${ }^{15}$ and T1WI is useful for SAH diagnosis in these phases.

Recently, several authors have reported that FLAIR and T2*weighted imaging sequences are the most sensitive and useful for detecting SAH in the acute and subacute phases. ${ }^{16-18}$ However, FLAIR and $\mathrm{T} 2{ }^{*}$-weighted imaging sequences are not useful for the diagnosis of the onset stage of the subarachnoid blood and cannot differentiate subarachnoid blood in the subacute and acute phases. As mentioned above, because subarachnoid blood is depicted clearly as BHSB on T1WI around 3 to 4 days after SAH onset, any BHSB that is detected by T1WI on admission is blood that is in the subacute phase (ie, occurred before admission). If there is a mismatch between the distribution of subarachnoid blood on T1WI and on FLAIR images, the blood depicted on FLAIR images but not T1WI is the more recent, new acute hemorrhage. This hemorrhage is the second hemorrhage (major attack) that resulted in hospitalization. Therefore, the BHSB detected by T1WI is in the subacute phase and must have occurred before admission. In this study, we interpreted this bleeding as having arisen from a minor leak.

While it is reasonable to label blood as subacute if it is hyperintense on T1WI, acute blood can also show increased T1 signal. ${ }^{13,19}$ Mitchell et al ${ }^{19}$ reported a sensitivity of $50 \%$ for T1 hyperintensity in the acute stage of SAH. However, their study was of a small sample $(n=20)$ and therefore included some patients in the subacute phase. In these studies, a strict evaluation of the timing of MR imaging relative to the onset of SAH is necessary. The acute phase of SAH is usually defined as within 72 hours of onset. In our study, we excluded patients with $\mathrm{SAH}$ who were admitted 48-72 hours after onset because this time window represents a period of oxyhemoglobin-to-methemoglobin conversion. We investigated MR imaging findings only in patients admitted within 48 hours of major SAH.

Subarachnoid blood can be very heterogeneous, and not all subacute blood will be T1 hyperintense. Many factors can affect these characteristics, including subarachnoid blood aging more slowly than cerebral hematomas (due to higher oxygen tension in
CSF) and likely different proportions of CSF and subarachnoid blood compounding the picture. From previous studies with small sample sizes, the sensitivity of T1 hyperintensity in the subacute stage of SAH ranged from $33 \%$ to $36 \% .{ }^{19,20}$ Bradley $^{21}$ reported that it was difficult to diagnose $\mathrm{T} 1$ hyperintensity in the subacute stage of SAH if there was an extremely small hemorrhage because the red blood cells are likely to have been resorbed by the time significant methemoglobin formation would have occurred. That a diagnosis of minor leak by T1-FLAIR mismatch and the evaluation of the exact staging of subarachnoid blood by the MR imaging has limitations is unavoidable.

Methemoglobin has high signal intensity on time-of-flight MRA and obscures signals from vessels and aneurysms. ${ }^{22,23} \mathrm{Ob}-$ fuscation of the visibility of a ruptured aneurysm on MRA becomes a simple neuroradiologic indicator of the presence of subarachnoid blood in the subacute phase due to a minor leak. Grandin et $\mathrm{al}^{23}$ reported that phase-contrast MRA could easily overcome these problems of obfuscation in the MRA. However, to limit the duration of the examination, we did not perform phase-contrast MRA.

\section{Minor Leaks in Elderly Patients with SAH}

From our results and those of a previous report, ${ }^{24}$ the incidence of a warning headache before a major attack was low in elderly patients with SAH. However, the frequency of potential minor leak according to T1-FLAIR mismatch was high in elderly patients with SAH. The causes of this result are unknown. We speculate that the age-associated enlargement of the subarachnoid space due to cortical atrophy may be 1 reason for the high incidence of potential minor leaks without warning headache in elderly patients. In other words, because an increase in intracranial pressure may not occur even in the presence of an aneurysmal rupture, symptoms due to minor leak would be milder in elderly patients than in younger patients with SAH. Therefore, it could be relatively rare that elderly patients with SAH go to the hospital at the time of the first attack due to minor leak, and they are often only brought to the hospital for a major SAH attack.

\section{Minor Leaks and ICH due to a Ruptured Aneurysm}

Several pathologic studies have reported that ICH due to a ruptured aneurysm occurs by indirect or direct rupture of an aneurysm in the brain. ${ }^{25-27} \mathrm{ICH}$ due to indirect rupture in the brain is caused by obstruction of the subarachnoid space due to the presence of blood, fibrin, and fibrous arachnoidal adhesions after previous bleeding. ${ }^{27}$ By contrast, $\mathrm{ICH}$ due to direct rupture into the brain occurs due to the adhesion of the aneurysm sac to the pia mater. ${ }^{25,26}$ One report also suggested that the adhesion of the aneurysm sac to the pia mater may be facilitated by previous bleeding. ${ }^{25}$ This possibility indicates that the likelihood of ICH due to aneurysm rupture is increased by the presence of a previous hemorrhage, such as a minor leak. Indeed, in our study, in all patients diagnosed with a minor leak and $\mathrm{ICH}$ on admission, the high-signal-intensity lesion on T1WI corresponded to subarachnoid blood in the neighboring area of the ruptured aneurysm. On the other hand, ICH was depicted as a high-signal-intensity lesion on 
FLAIR images and DWI on admission and did not show high signal intensity on T1WI. These findings suggest that the ICH present on admission was a new, acute lesion.

\section{Minor Leaks and Rebleeding}

Previous reports have indicated that a warning headache is a risk factor for rebleeding, because a fragile aneurysm that ruptured at the time of the minor leak is prone to rebleeding. ${ }^{28,29}$ Additionally, existing literature indicates that the rate of rebleeding is higher in patients with $\mathrm{ICH}$ than in patients without $\mathrm{ICH}{ }^{29,30}$ Our results and previous reports suggest that a minor leak is related closely to $\mathrm{ICH}$ and rebleeding. Therefore, in patients who experienced a minor leak before the major SAH, emergency surgery should be performed as soon as possible because these patients have a high probability of both rebleeding and ICH. ${ }^{28,29}$

\section{Minor Leak and Prognosis}

Unexpectedly, several previous studies have reported that the presence of a warning leak had no impact on outcome. ${ }^{24,31}$ In these studies, the true outcome of patients with SAH with a minor leak was difficult to establish because of the difficulty of obtaining complete information on the presence or absence of a warning headache by interview. Jakobsson et $\mathrm{al}^{1}$ emphasized that outcomes were particularly unfavorable in patients with a short interval ( $\leq 3$ days) from interview-diagnosed warning headache to $\mathrm{SAH}$. Although our neuroradiologic diagnostic procedure for a minor leak cannot be used to identify patients who had $<2$ days between minor leak and major $\mathrm{SAH}$, our results, combined with those of Jakobsson et al, ${ }^{1}$ emphasize that the prognosis of patients with SAH with a minor leak before a major attack is unfavorable.

\section{Limitations}

In patients with an extremely small amount of subacute blood, the sensitivity of the diagnosis of T1 hyperintensity may decrease. In addition, this neuroradiologic diagnostic procedure cannot be used to diagnose minor leaks in patients admitted $>48$ hours after major SAH attack. Similarly, this procedure cannot be used to diagnose minor leaks in patients who had major SAH within 48 hours of minor leak onset. It is difficult to distinguish between patients with preceding headache due to the occurrence of dissection without $\mathrm{SAH}^{32}$ and patients with minor leak (SAH) due to ruptured dissecting aneurysms. Therefore, we excluded patients with dissecting cerebral aneurysms from this study.

\section{CONCLUSIONS}

In this study, we have proposed a method to define patients who had a prior minor leak: T1-detected BHSB on admission MR imaging was considered subacute, and FLAIR hyperintense blood that was more extensive than this was deemed the acute component. The T1 hyperintense component was defined as a subacute minor leak. We found that almost all patients with warning headache diagnosed by interview had a minor leak according to our definition. We conclude that warning headaches are not a product of recall bias but are the result of actual leaks.

\section{REFERENCES}

1. Jakobsson KE, Saveland H, Hillman J, et al. Warning leak and management outcome in aneurysmal subarachnoid hemorrhage. J Neurosurg 1996;85:995-99

2. Linn FH, Wijdicks EF, van der Graaf Y, et al. Prospective study of sentinel headache in aneurysmal subarachnoid haemorrhage. Lancet 1994;344:590-93

3. Gillingham FJ. The management of ruptured intracranial aneurysms. Scott Med J 1967;12:377-83

4. Drake CG. Progress in cerebrovascular disease: management of cerebral aneurysm. Stroke 1981;12:273-83

5. Linn FH, Rinkel GJ, Algra A, et al. The notion of "warning leaks" in subarachnoid haemorrhage: are such patients in fact admitted with a rebleed? J Neurol Neurosurg Psychiatry 2000;68:332-36

6. Drake CG. Report of World Federation of Neurological Surgeons Committee on a Universal Subarachnoid Hemorrhage Grading Scale. J Neurosurg 1988;68:985-86

7. Fisher CM, Kistler JP, Davis JM. Relation of cerebral vasospasm to subarachnoid hemorrhage visualized by computerized tomographic scanning. Neurosurgery 1980;6:1-9

8. Shinohara Y, Minematsu K, Amano T, et al. Modified Rankin scale with expanded guidance scheme and interview questionnaire: interrater agreement and reproducibility of assessment. Cerebrovasc Dis 2006;21:271-78

9. Shimoda M, Hoshikawa K, Shiramizu H, et al. Early infarction detected by diffusion-weighted imaging in patients with subarachnoid hemorrhage. Acta Neurochir (Wien) 2010;152:1197-205

10. Hauerberg J, Andersen BB, Eskesen V, et al. Importance of the recognition of a warning leak as a sign of a ruptured intracranial aneurysm. Acta Neurol Scand 1991;83:61-64

11. Spickler E, Lufkin R, Teresi L, et al. MR imaging of acute subarachnoid hemorrhage. Comput Med Imaging Graph 1990;14:67-77

12. Ogawa T, Inugami A, Shimosegawa E, et al. Subarachnoid hemorrhage: evaluation with MR imaging. Radiology 1993; 186:345-51

13. Bradley WG Jr, Schmidt PG. Effect of methemoglobin formation on the MR appearance of subarachnoid hemorrhage. Radiology 1985;156:99-103

14. Wahlgren NG, Lindquist C. Haem derivatives in the cerebrospinal fluid after intracranial haemorrhage. Eur Neurol 1987;26:216-21

15. Ogawa $\mathrm{T}$, Inugami A, Fujita $\mathrm{H}$, et al. MR diagnosis of subacute and chronic subarachnoid hemorrhage: comparison with CT. AJR Am J Roentgenol 1995;165:1257-62

16. Shimoda M, Hoshikawa K, Shiramizu H, et al. Problems with diagnosis by fluid-attenuated inversion recovery magnetic resonance imaging in patients with acute aneurysmal subarachnoid hemorrhage. Neurol Med Chir (Tokyo) 2010;50:530-37

17. Noguchi K, Seto H, Kamisaki Y, et al. Comparison of fluid-attenuated inversion-recovery MR imaging with CT in a simulated model of acute subarachnoid hemorrhage. AJNR Am J Neuroradiol 2000;21:923-27

18. Imaizumi $\mathrm{T}$, Chiba $\mathrm{M}$, Honma $\mathrm{T}$, et al. Detection of hemosiderin deposition by $\mathrm{T} 2{ }^{\star}$-weighted MRI after subarachnoid hemorrhage. Stroke 2003;34:1693-98

19. Mitchell P, Wilkinson ID, Hoggard N, et al. Detection of subarachnoid haemorrhage with magnetic resonance imaging. $\mathrm{J} \mathrm{Neurol} \mathrm{Neu-}$ rosurg Psychiatry 2001;70:205-11

20. Noguchi K, Ogawa T, Seto H, et al. Subacute and chronic subarachnoid hemorrhage: diagnosis with fluid-attenuated inversion-recovery MR imaging. Radiology 1997;203:257-62

21. Bradley WG Jr. MR appearance of hemorrhage in the brain. Radiology 1993;189:15-26

22. Ida M, Kurisu Y, Yamashita M. MR angiography of ruptured aneurysms in acute subarachnoid hemorrhage. AJNR Am J Neuroradiol 1997; 18:1025-32 
23. Grandin CB, Cosnard G, Hammer F, et al. Vasospasm after subarachnoid hemorrhage: diagnosis with MR angiography. AJNR Am J Neuroradiol 2000;21:1611-17

24. Okawara $\mathrm{SH}$. Warning signs prior to rupture of an intracranial aneurysm. J Neurosurg 1973;38:575-80

25. Tomlinson BE. Brain changes in ruptured intracranial aneurysm. J Clin Pathol 1959;12:391-99

26. Crompton MR. Intracerebral haematoma complicating ruptured cerebral berry aneurysm. J Neurol Neurosurg Psychiatry $1962 ; 25: 378-86$

27. Robertson EG. Cerebral lesions due to intracranial aneurysms. Brain 1949;72:150-85

28. Beck J, Raabe A, Szelenyi A, et al. Sentinel headache and the risk of rebleeding after aneurysmal subarachnoid hemorrhage. Stroke 2006;37:2733-37

29. Güresir E, Beck J, Vatter H, et al. Subarachnoid hemorrhage and intracerebral hematoma: incidence, prognostic factors, and outcome. Neurosurgery 2008;63:1088-93; discussion 1093-94

30. Naidech AM, Janjua N, Kreiter KT, et al. Predictors and impact of aneurysm rebleeding after subarachnoid hemorrhage. Arch Neurol 2005;62:410-16

31. Juvela S. Minor leak before rupture of an intracranial aneurysm and subarachnoid hemorrhage of unknown etiology. Neurosurgery 1992;30:7-11

32. Mizutani T. Natural course of intracranial arterial dissections. J Neurosurg 2011;114:1037-44 